\title{
Modelling Considerations for Resistance Wire Thermometers Applied to Internal Combustion Engines
}

\author{
Varun Venkataraman ${ }^{1}$, Yushi Murai², \\ Michael Liverts' ${ }^{2}$ Ramis Örlü2, Jens H.M. Fransson², Ola Stenlåås ${ }^{1,3}$, Andreas Cronhjort ${ }^{1}$ \\ ${ }^{1}$ CCGEx, Dept. Machine Design, KTH Royal Institute of Technology, 10044 Stockholm, Sweden \\ ${ }^{2}$ CCGEx, Dept. Engineering Mechanics, KTH Royal Institute of Technology, \\ 10044 Stockholm, Sweden \\ ${ }^{3}$ Scania CV AB, 15187 Södertälje, Sweden \\ Varun Venkataraman: varunve@kth.se
}

\begin{abstract}
Summary:
This study delves into the modelling of resistance wire thermometers (RWTs) within the application context of measuring the exhaust gas temperature pulse in internal combustion engines. The model was developed in a commercial simulation software utilizing the heat balance equation. Disparities were found between different model representations of the prongs due to differences in the heat transfer within the sensor, which impacts its expected dynamic response. The appropriate modelling choice will be made upon validation with shock tube experiments for different RWT designs.
\end{abstract}

Keywords: cold-wire anemometry, lumped parameter, resistance thermometry, time-resolved temperature measurement

\section{Background, Motivation and Objective}

Measurements of the time-resolved exhaust gas flow in internal combustion engines (ICEs) remains complex and challenging. As ICEs are expected to play a significant role in the broader effort to curb climate change, continuous and often radical improvements are demanded on its efficiency and emission mitigation levels [1]. As temperature is a crucial parameter to meet this objective, its measurement on a timeresolved basis would aid in the further development of turbocharging, waste heat recovery and aftertreatment systems.

A recent study indicated the potential to address the challenge of measuring the timeresolved exhaust gas temperature pulse in ICEs using specially designed resistance wire thermometers (RWTs) [2]. An outcome of this preliminary assessment was the need to characterise the dynamic behaviour of the RWTs based on its construction and application environment. This study initiates the assessment using models to complement experimental investigations.

Modelling of RWTs is a well-studied topic primarily concerned with resolving the heat loss from the sensing wire to the prongs to account for end conduction heat losses. These models provide a design guideline for RWTs to minimise end conduction and can be used for dynamic response correction through the frequen- cy response function of the sensor system. While literature in this topic is expansive, two particular modelling approaches are currently examined.

Model-1 developed in Ref. [3] used the lumped parameter approach of heat transfer modelling wherein each element of the RWT (wire, stub and prongs) was lumped as a single element. Model-2 developed in Ref. [4] represented the RWT as a weighted sum of first-order systems representing the sensing wire and the prongs discretized into four distinct elements. Hence, the two models differ primarily in their representation of the prongs.

In this study, the implications of the modelling approach proposed in model-1 and model-2 are evaluated on the response of an RWT to a simulated flow profile.

\section{Sensor Modelling Methodology}

One of the RWTs analysed in Ref. [4] was modelled in the powertrain oriented system simulation software, GT-Power. Fig.1 illustrates a schematic of the modelled RWT along with the modelling approaches. When the prongs are identical, it is sufficient to model one-half of the sensor system due to assumed symmetry over the wire. The Biot number (Bi) of the element determines the validity of the lumped parameter approximation. It is defined by the ratio of the conductive to convective thermal re- 
sistance. $\mathrm{A} \mathrm{Bi} \ll 1$ provides a better lumped parameter approximation of the modelled system. An additional thermal resistance is included to account for the unexposed length of the prongs which act as a heat sink. All of the exposed lumped elements of the RWT experience a forced convective component from the flow in addition to the conduction between them. The effect of radiation was insignificant and not included in the current evaluation.

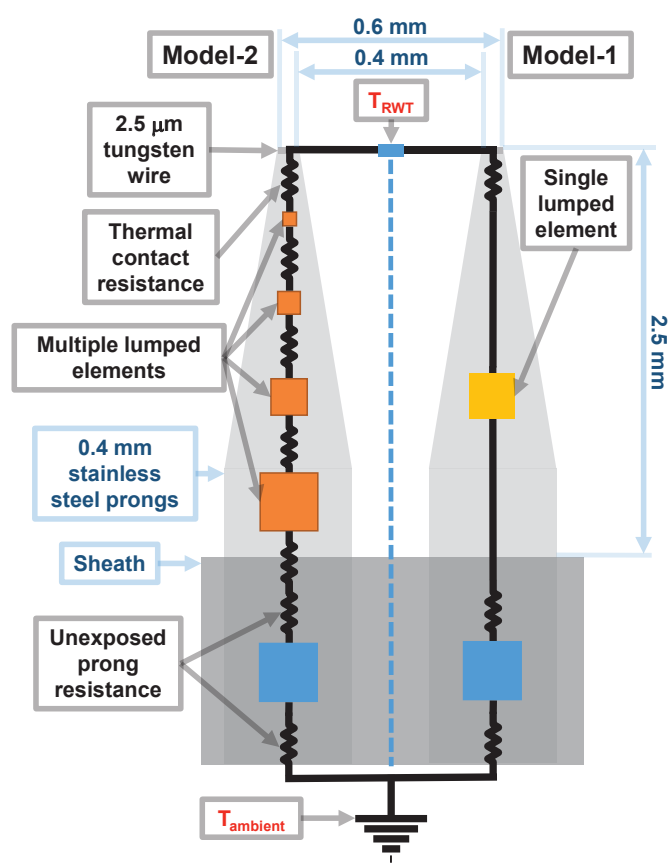

Fig. 1. Sensor schematic and modelling approach

While the overall probe dimensions were available in Ref. [4], approximations were made on dimensions and material properties not explicitly stated in the study. This especially applies to the thermal contact resistance induced by the joining process between the sensing wire (tungsten) and the prongs (stainless steel). The model-1 study [3] states a thermal contact resistance of the order of $50000 \mathrm{~K} / \mathrm{W}$ although it was derived for soldering of a $50 \mu \mathrm{m}$ silver stub onto conical stainless steel prongs of root diameter between 0.3-0.4 mm. Despite the wire being welded in the modelled sensor and not containing a stub, the thermal contact resistance suggested in Ref. [3] was used to assess the modelling approaches. The contact (weld) area between the wire and prong along with the prong mass were constant between the modelling approaches.

\section{Implications of the Modelling Approach}

To ascertain the sensitivity of RWT modelling, the experimental setup used in Ref. [4] was modelled. The temperature and velocity over the RWT were increased as a ramp depicted in the inset of Fig. 2. The ramp represents a sensor immersed in a heated jet at $0.5 \mathrm{~m} / \mathrm{s}$ from the ambient condition. The Biot number criterion was valid for elements in both modelling approaches over the ramp. Fig. 2 shows the simulated response based on the two models. While the initial portion of the response of the sensing wire is comparable between the approaches, the attenuation imposed by end conduction to the prong is more severe in model- 1 . This is an expected observation from a theoretical viewpoint as a larger lumped element interacts directly with the sensing wire in model-1. Additionally, model-2 accounts for the length scale of the exposed prong with four lumped elements of varying size, unlike model-1.

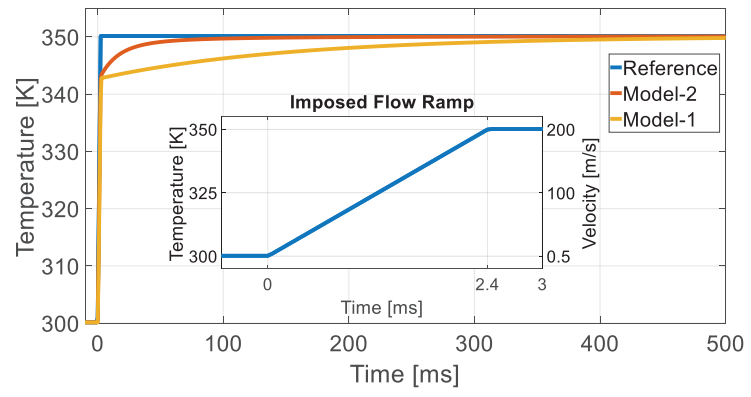

Fig. 2. Modelled RWT wire response to flow ramp

\section{Conclusion}

Despite the validity of the lumped parameter approach, differences were observed in the RWT dynamic response based on the choice of prong modelling. Experiments of RWTs with different design features in a shock tube will help validate the appropriate modelling choice associated with the prongs. The derived understanding can subsequently be extended to more complex flows as that of an ICE exhaust.

\section{Acknowledgements}

This study was financed by the Swedish Energy Agency and the Competence Center for Gas Exchange (CCGEx).

\section{References}

[1] J. R. Serrano et al., Why the development of internal combustion engines is still necessary to fight against global climate change from the perspective of transportation, Appl. Sci., 9, 4597 (2019), doi: 10.3390/app9214597.

[2] V. Venkataraman et al., Resistance Wire Thermometers for Temperature Pulse Measurements on Internal Combustion Engines, in SMSI2020, 252-253, (2020) doi: 10.5162/SMSI2020/P2.14.

[3] G. Arwatz et al., Dynamic calibration and modeling of a cold wire for temperature measurement, Meas. Sci. Technol., 24, 125301 (2013), doi: 10.1088/0957-0233/24/12/125301.

[4] R. Dénos, C. H. Sieverding, Assessment of the cold-wire resistance thermometer for high-speed turbomachinery applications, J. Turbomach., 119, 140-148 (1997), doi: 10.1115/1.2841002. 